\title{
AI-based Brain Image Segmentation Using Synthesized Data
}

Pouya Tavousi ${ }^{1}$, zahra Shahbazi $^{2}$ and Sina Shahbazmohamadi ${ }^{3}$

${ }^{1}$ University of Connecticut, Storrs, Connecticut, United States, ${ }^{2}$ Manhattan College, Storrs, Connecticut, United States, ${ }^{3}$ REFINE lab/ University of Connecticut, Storrs, Connecticut, United States

The success of connectomics research in mapping the neural interconnections, as needed for understanding brain functions, largely depends on advances in microscopy and image analysis. Miniature features in brain rule its functions. Yet, large regions of interest (ROIs) must be scouted, for a proper understating of neural network system. This requires obtaining high-resolution tomographies of relatively large brain samples [1]. Regardless of what tomography technique is used, a critical step is the segmentation of the produced images to identify the boundaries of the constituent objects within the brain sample of interest. For that, each pixel (voxel) in the 2D (3D) image is assigned a label that is shared among all the pixels (voxels) of the same object, resulting in a 2D (3D) image that is partitioned into several groups of connected pixels (voxels) [2]. Doing so is necessary for follow-up interpretation steps but it faces significant challenges. Conventionally, the segmentation process is conducted manually, where trained individuals spend months to segment volumes in the order of cubic millimeters [1]. Aside from extensive labor and cost involved, such manual process entails human error. Double-checking and triplechecking practices to eliminate such error would add to the required time and effort for conducting the manual segmentation. Existing attempts to crowdsource the manual segmentation task using volunteers face the challenge of volunteer's lack of motivation [3]. On top of these, a major drawback of the stateof-the-art software packages that enable manual segmentation is that the user, at each point in time, has only a 2D perception of the brain sample, which acts as a prohibitive factor on the way of conducting a realistic segmentation. Automated segmentation techniques tend to address these issues but they come short of providing a reliable solution [4]. Conventional computer-vision-based segmentation methods, such as thresholding are often only useful for aiding the manual segmentation and the machine-learningbased segmentation algorithms [5] suffer from shortage of ground truth data, especially because the required data for supervised training of these algorithms must be produced manually.

We propose a model-based approach to develop a fully automated segmentation method for 3D brain images, from different modalities. For that, we will leverage simulated imaging algorithms to produce labeled data for training machine-learning algorithms. Different classes of neurons will be considered. For each class, first a detailed computer aided design (CAD) model of the neuron, so called as "virtual" neuron, will be constructed, by reverse-engineering high-resolution tomographies (i.e. 3D images) of real brain samples. Next, the image of each neuron will be simulated (at different microscopy modalities), using physics-based image simulator algorithms. These algorithms will take the computer model of the neuron and the spatial map of its chemical composition, as well as a set of imaging parameters to generate a highly accurate image of the neuron in silico. Throughout an iterative process, the image simulation settings will be fine-tuned by conducting a quantitative comparison between the simulated image of the virtual neuron and the real image of the real neuron, through a feedback loop. This is followed by parametrizing the geometry of each neuron class to allow for an efficient, yet realistic, enumeration of different instance of each class of neuron. Then, through a combinatorial algorithm, a large number (thousands, millions or billions) of virtual, yet realistic, brain neural network blocks are generated in silico, where each block represents a different arrangement of virtual neurons and mimics a real brain sample block. For this, the geometric parameters, which define the shape of the a neuron, as well as the pose parameters, which define its orientation and position in space, will be set, using a random sampling approach, to generate different 
virtual neurons. In fact, in each random selection, the geometric and pose parameters will adopt values within a plausible range. Also, material composition distribution of the neuron will be determined, through a random sampling approach and using the information at hand about the plausible chemical composition of each class of neurons. This is followed by putting the generated virtual neurons together to form virtual brain sample blocks. Although virtual, this brain block will contain a realistic network of neurons of various classes, sizes, shapes and orientations. This process is repeated until sufficient number of virtual brain blocks are produced in silico. Each virtual brain block will then undergo a simulated imaging step. Artefacts such as noise and charging will be incorporated in the simulation to result in more realistic images. The pair of the resulting in silico image and the in silico brain block is expected to serve as a highly realistic ground truth dataset for training the machine-learning algorithm for automated segmentation. One key advantage of the proposed segmentation method is that, it leverages the context (i.e., the knowledge that the objects are neurons) for a significantly more effective segmentation. That is, by narrowing down the set of possible geometries that could have resulted the image, reverse-engineering of the images to obtain the corresponding neural network is significantly facilitated. Further, due to the fact, in theory, there is no limitation in terms of the number of generated training data, the proposed approach is expected to significantly outperform currently practiced machine learning methods that use human-produced labeled data from real samples for training purposes.

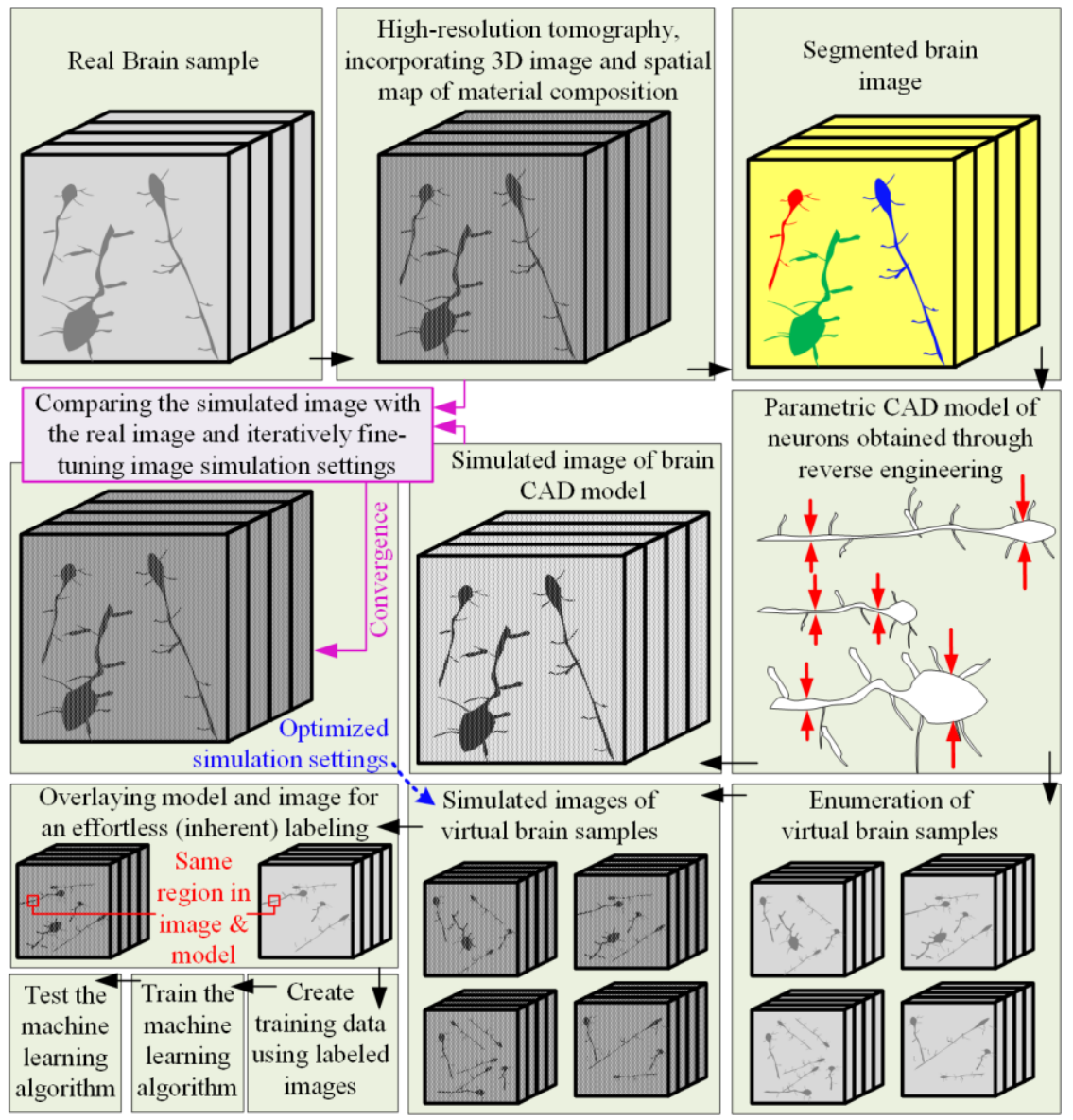

Figure 1. Schematic overview of the proposed innovation for establishing an automated brain segmentation method 


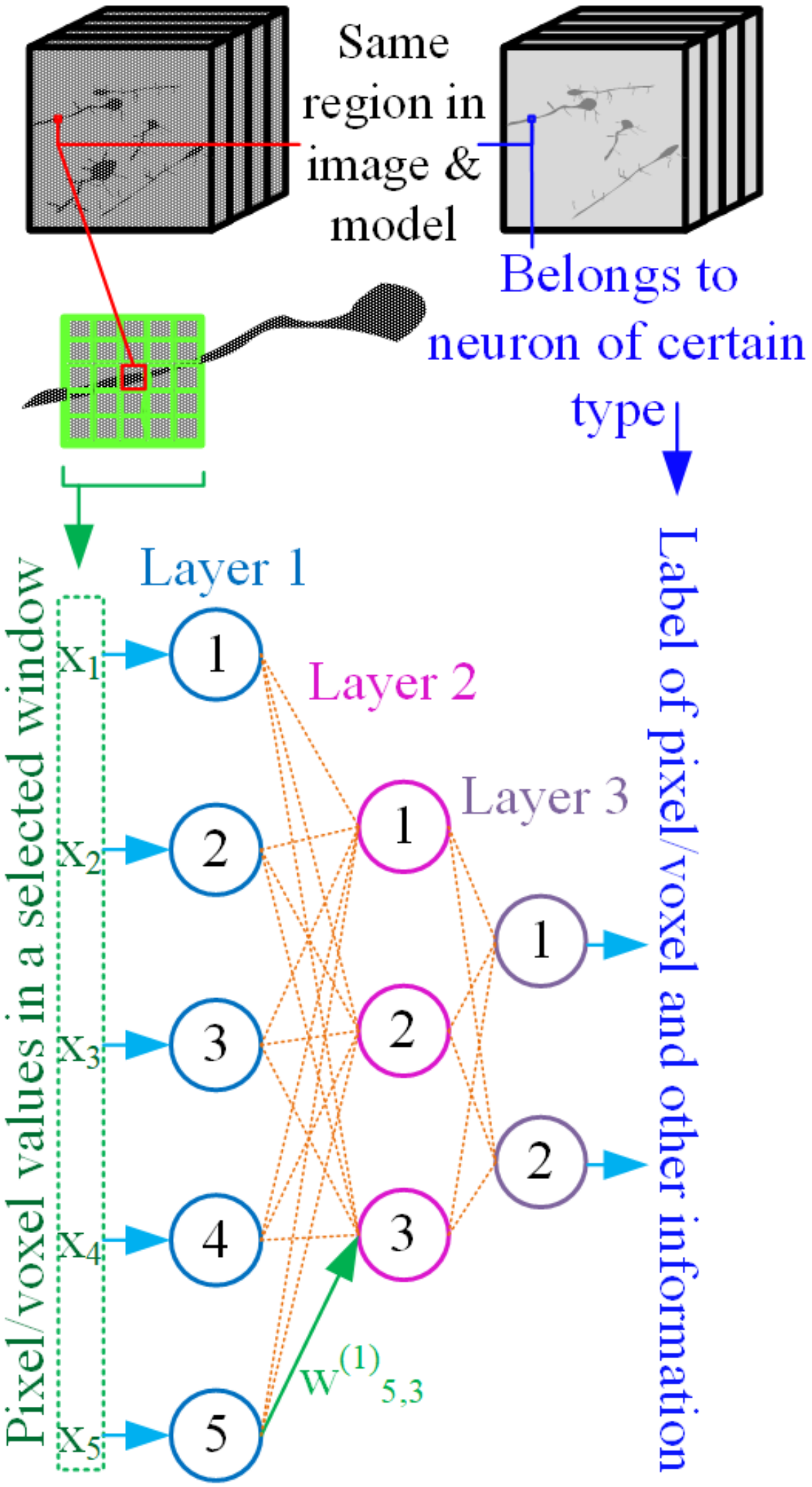

Figure 2. Artificial neural network (ANN)'s generic architecture used for automatic labeling of the pixels/voxels of brain images 


\section{References}

[1] N. Kasthuri, K. J. Hayworth, D. R. Berger, R. L. Schalek, J. A. Conchello, S. Knowles-Barley, D. Lee, A. Vázquez-Reina, V. Kaynig, T. R. Jones and others, "Saturated reconstruction of a volume of neocortex," Cell, vol. 162, pp. 648-661, 2015.

[2] N. M. Zaitoun and M. J. Aqel, "Survey on image segmentation techniques," Procedia Computer Science, vol. 65, pp. 797-806, 2015.

[3] I. Arganda-Carreras, S. C. Turaga, D. R. Berger, D. Cireşan, A. Giusti, L. M. Gambardella, J. Schmidhuber, D. Laptev, S. Dwivedi, J. M. Buhmann and others, "Crowdsourcing the creation of image segmentation algorithms for connectomics," Frontiers in neuroanatomy, vol. 9, p. 142, 2015.

[4] A. Vazquez-Reina, M. Gelbart, D. Huang, J. Lichtman, E. Miller and H. Pfister, "Segmentation fusion for connectomics," in 2011 International Conference on Computer Vision, 2011.

[5] Q. Rao, H. Han, W. Li, L. Shen, X. Chen and Q. Xie, "Automatically segmenting and reconstructing neurons in SEM images," in 2016 IEEE International Conference on Mechatronics and Automation, 2016. [6] I. Cabezón, E. Augé, M. Bosch, A. J. Beckett, I. A. Prior, C. Pelegr'i and J. Vilaplana, "Serial blockface scanning electron microscopy applied to study the trafficking of 8D3-coated gold nanoparticles at the blood--brain barrier," Histochemistry and cell biology, vol. 148, pp. 3-12, 2017.

[7] P. Westenberger, "AVIZO-3D visualization framework," in Geoinformatics Conference, 2008.

[8] R. Makovetsky, N. Piche and M. Marsh, "Dragonfly as a Platform for Easy Image-based Deep Learning Applications," Microscopy and Microanalysis, vol. 24, pp. 532-533, 2018.

[9] A. P. Srikanthan and others, "REVERSE ENGINEERING OF MACHINE PART BY PARAMETRIC MANNER," 2019.

[10] J. Favata and S. Shahbazmohamadi, "Realistic non-destructive testing of integrated circuit bond wiring using 3-D X-ray tomography, reverse engineering, and finite element analysis," Microelectronics Reliability, vol. 83, pp. 91-100, 2018. 